\title{
Entretien réalisé par Gabrielle Houbre, Christiane Klapisch-Zuber et Pauline Schmitt-Pantel
}

\section{Denyse DURAND-RUEL}

\section{(2) OpenEdition \\ 1 Journals}

Édition électronique

URL : http://journals.openedition.org/clio/654

DOI : 10.4000/clio.654

ISSN : 1777-5299

Éditeur

Belin

Édition imprimée

Date de publication : 1 avril 2004

Pagination : 169-179

ISSN : 1252-7017

\section{Référence électronique}

Denyse DURAND-RUEL, «Entretien réalisé par Gabrielle Houbre, Christiane Klapisch-Zuber et Pauline Schmitt-Pantel », Clio. Femmes, Genre, Histoire [En ligne], 19 | 2004, mis en ligne le 13 novembre 2006, consulté le 03 mai 2019. URL : http://journals.openedition.org/clio/654; DOI : 10.4000/clio.654

Ce document a été généré automatiquement le 3 mai 2019.

Tous droits réservés 


\title{
Entretien réalisé par Gabrielle Houbre, Christiane Klapisch-Zuber et Pauline Schmitt-Pantel
}

\author{
Denyse DURAND-RUEL
}

1 Denyse et Philippe Durand-Ruel commencent leur collection de tableaux et d'objets d'art au début des années soixante : ils héritent alors d'une partie de la collection de tableaux impressionnistes qui avait été constituée par l'arrière-grand-père de Philippe Durand-Ruel. Ils décident de poursuivre cette collection. Leur intention première était de faire le lien entre le temps des impressionnistes et l'époque contemporaine, mais ils se rendent rapidement compte que des peintres comme Picasso ou Matisse avaient acquis une telle valeur qu'il leur aurait fallu vendre deux impressionnistes pour acheter un Picasso, par exemple. Ils font nt.

2 Denyse et Philippe Durand-Ruel ont deux fils, dont l'un dirige le département d'art contemporain chez Christie's.

$\mathrm{Q}$ Dites-nous comment vous avez commencé votre collection de tableaux.

$4 \quad \mathrm{R}$ Au début des années soixante nous avons d'abord connu Sami Tarika qui nous a beaucoup appris sur l'art contemporain, puis Michel Couturier chez qui nous avons trouvé les œuvres de Wols et de Fautrier dont nous avons fait la connaissance et que nous avons pas mal collectionné, encore de son vivant. Ensuite, nous avons commencé avec les artistes contemporains, c'est-à-dire les Nouveaux Réalistes de l'École de Nice, donc Arman, César, Klein... À cette époque-là, cela a été vraiment mon mari qui a lancé la collection parce qu'il avait un ami aussi passionné que lui, Jean-Marie Rossi, qui était antiquaire (de préférence du XVIIIe siècle). Ils se sont d'ailleurs très vite associés pour le magasin d'antiquités et ils parcouraient toutes les galeries ensemble comme des fous. On a encore collectionné Schwitters, ses peintures entre 1920 et 1940. Mais, à part ça, c'est vraiment l'art contemporain qui nous a passionnés.

Q Et en même temps, vous avez ouvert une galerie ? 
6 R Non, nous ne nous sommes jamais lancés dans le commerce de l'art. La galerie qui portait le nom de Durand-Ruel et qui était gérée par l'oncle de mon mari a fermé il y a plus de vingt ans.

Q Et vous, quand avez-vous eu l'idée de constituer des archives des peintres ?

R J'ai commencé dès 1968, nous sommes beaucoup sortis avec les artistes et je me suis rendu compte qu'ils n'avaient aucune archive. Ils faisaient des œuvres, ils avaient des catalogues, mais rien n'était répertorié, classé. Je me suis proposée, bénévolement, car je n'avais pas besoin d'argent pour vivre, pour leur tenir leurs archives. Et j'ai commencé en fait avec deux jeunes, qui sont Jean-Pierre Raynaud et Jean-Claude Farhi, après j'ai laissé Jean-Claude Farhi parce qu'il travaillait dans le Midi et que ce n'était pas pratique. Et làdessus, César me voyant travailler, au bout de six mois est venu me voir et me dit : «Écoute, je suis de l'âge d'être le père de Jean-Pierre Raynaud, tu pourrais t'occuper de moi d'abord, avant de prendre un petit jeune ». Je lui dis : «Si tu veux, je veux bien », et Arman qui est toujours en compétition avec César a dit : «Il n'y a aucune raison que tu t'occupes de César et pas de moi ». Alors je me suis retrouvée avec les trois et j'ai ajouté Bertrand Lavier. C'est de la folie raide et je travaille sans arrêt, sans arrêt.

$\mathrm{Q}$ En quoi consiste votre travail d'archives?

10 R Cela a consisté, par exemple pour César que j'ai pris quand il avait déjà une belle carrière derrière lui, à aller voir tous les photographes qui avaient fait des photos de César, j'ai acheté toutes les photos qu'ils avaient faites, et puis je me suis mise à les classer, avec les catalogues et avec son aide à lui. Et puis j'ai fait des bouquins, j'ai commencé à éditer son catalogue raisonné : le premier tome est sorti. Arman, j'en ai fait deux tomes, et Jean-Pierre Raynaud un seul, et je suis en train de travailler sur le suivant.

Q Archivez-vous également les documents écrits ?

12 R J'archive bien sûr leur presse, tout ce qui a été écrit et publié sur eux et sur leur œuvre ! Mais ils écrivent rarement eux-mêmes. César par exemple n'a jamais écrit ; il a donné des interviews - d'ailleurs il parlait très bien - mais il n'écrivait jamais.

Q Et les contrats?

14 R Non, non, je ne suis pas du tout du côté des affaires et je ne m'occupe absolument pas des prix. Quand quelqu'un me demande : «Vous savez combien je pourrais vendre tel tableau ", je dis : "Je n'en ai pas la moindre idée ». C'est complètement en dehors de mes attributions et de ce qui m'intéresse. Je ne gère que les images, la vie des œuvres.

$\mathrm{Q}$ Et concrètement, comment tenez-vous ces archives ? faite à la main avec sa photo, la liste de tous les lieux où elle a été exposée et la liste de tous les livres ou revues où elle est reproduite.

R J'ai commencé avec les Editions de la Différence, et puis j'ai eu de gros problèmes avec eux parce qu'ils ont dépensé l'argent donné par les artistes pour l'édition des catalogues raisonnés pour régler leurs dettes !!! Pour Jean-Pierre Raynaud, je travaille avec les Éditions du Regard qui sont parfaites et, heureusement, Arman vient de retrouver un jeune éditeur italien.

Q Et n'aimeriez-vous pas éditer vous-même?

R Non, c'est déjà un travail de titan, je n'aurais jamais dû prendre quatre artistes. 

mais moi j'ai un peu plus besoin de réfléchir, de comparer. Mais je suis aussi très fière de certains de mes achats. Par exemple, dans une vente, au début des années 80 , il y a un tableau qui me plaît beaucoup, un type que je ne connaissais pas du tout, il s'appelait Basquiat, je l'ai eu pour rien du tout, $60000 \mathrm{f}$., quelque chose comme ça. On le sent si c'est costaud, si c'est quelqu'un qui innove. Même quelqu'un dont on n'a jamais entendu parler, on le repère. 
R Oui, dernièrement, j'ai par exemple acheté un rhinocéros de Xavier Veilhan et une œuvre de Emmanuel Saulnier. Et puis je complète la collection que nous avons de JeanLuc Parant que j'aime beaucoup. Mon fils, qui est un passionné d'art contemporain - et qui s'y connaît beaucoup mieux que moi - collectionne beaucoup, achète beaucoup.

Q Y-a-t-il une différence entre un collectionneur et une collectionneuse?

R Mon mari est carrément un collectionneur total dans l'âme, c'est-à-dire que n'importe quoi, il est prêt à en commencer une collection, n'importe quoi : pipes, bouquins, chapeaux... Il garde absolument tout, moi non, non, non, moi, je largue, évidement pas les tableaux... Nous en avons échangé quelques-uns, par exemple avec Arman, des tableaux anciens contre des œuvres plus contemporaines.

$\mathrm{Q}$ À partir d'un certain moment, vous êtes devenue une référence ?

$\mathrm{R}$ Non, mais tout le monde sait que je connais bien, j'ai été pendant des années et des années à la commission d'achat du Musée national d'art moderne à Beaubourg, on y apprend beaucoup et c'est follement intéressant.

Q Y a-t-il beaucoup de femmes dans ce milieu?

R Oui, oui, nous avons eu une grande mécène, au Musée national d'art moderne, Sylvie Boissonnas qui s'y connaissait merveilleusement bien. Dans les jeunes galeries, il y a beaucoup de femmes, auparavant c'était plutôt des hommes.

Q Avez-vous le même regard sur les tableaux que votre mari ?

R J'ai un regard un peu plus pointu, à mon avis ; lui y va par coup de cœur, il peut en acheter trois quatre de la même facture, de la même série, moi je dis : «non, un suffit, regarde, si on prenait plutôt celui-là, ce serait différent, ils se complèteraient ».

$Q$ Vous êtes donc plus rationnelle et votre mari plus sentimental ?

R Exactement. Lui est un vrai collectionneur, il aime amasser et collectionner est pour lui une passion. Moi, pas du tout. C'est vraiment choisir et garder ce qui est le mieux à mes yeux qui me passionne.

Q Il vous arrive de revendre?

R Non, nous n'avons jamais revendu.

$Q$ Vous vous concertez avec votre mari?

R Les grands tableaux, je lui demande toujours, il y a l'aspect financier, et j'aime être confortée dans mon jugement ; les petites choses, je les achète moi-même sans problème.

Q Vous passez beaucoup de temps dans les expositions?

R Oui, énormément, nous ne sortons plus que dans ce milieu-là. J'étais il y a trois jours à Londres pour aller voir la Foire et puis la grande exposition de Polke à la Tate. Je m'occupe aussi des Amis du Musée national d'art moderne, avec François Trêves et nous faisons beaucoup de voyages, de visites d'expositions avec les commissaires qui les ont préparées, ce qui nous apprend énormément, des visites d'ateliers avec les artistes. Et puis nous faisons aussi de vrais voyages, l'an dernier nous sommes allés quinze jours au Brésil pour voir toutes les collections, cette année huit jours au Portugal.

51 Q Vous achetez beaucoup à l'étranger ?

R Non, plutôt en France.

Q Un problème que nous connaissons bien avec les bouquins : de combien d'espace faut-il disposer pour faire une collection? 

actuel du Musée national d'art moderne, avait organisé un colloque au moment de «Passions privées », la grande exposition sur les collectionneurs au Musée d'art moderne de la Ville de Paris, colloque auquel il m'avait demandé de participer. C'était une tableronde, et la veille au soir Alfred me dit : «Bon, toi tu parles à telle heure », et je réponds : «Comment, moi je parle ? il n'en est même pas question, je suis incapable de parler devant une salle ». Et j'avais l'impression que ceux qui avaient parlé avant moi avaient déjà tout dit sur les collectionneurs. Alors, en y pensant et en cherchant que dire pendant la nuit, je me suis dit : «Je vais parler de la femme du collectionneur ». Et j'ai commencé mon speech comme ça : «Moi, je vais vous raconter la vie terrible de la femme du collectionneur ». Et j'ai raconté, en riant bien sûr, comment dans mon appartement à Paris, les meubles se sont mis à avancer, parce que derrière les canapés il y avait des tableaux, et cela de chaque côté, si bien que quand on s'asseyait sur les fauteuils on avait les genoux coincés contre la table, l'entrée de l'appartement qui était grande était devenue juste un petit couloir parce qu'elle était pleine de tableaux. Déménager devenant indispensable, nous avons cherché un grand loft dans le 14e, une vieille usine, qu'on aurait transformée. Nous n'avons pas trouvé, mais une amie nous a trouvé cette maison. Je détestais l'idée d'habiter en banlieue. Je ne connaissais rien aux fleurs et il y avait un immense jardin. Les enfants ont dû partir en pension car les travaux n'en finissaient pas. Bref, tout cela à cause de la collection. Vous voyez la vie épouvantable de la femme d'un collectionneur.

Q Jusqu'au jour où la femme du collectionneur s'est dit : après tout, je vais devenir actrice.

R Oh ! mais je l'étais déjà !

Q Vos enfants auraient pu être dégoûtés. C'est difficile de cohabiter avec tant d'œuvres d'art...

R Oui, bien sûr. Mais les enfants ont été plongés là-dedans depuis leur naissance. Il y a un déjeuner à la maison en général tous les dimanches, et il n'y a que des amis de l'art et des artistes qui sont là. Un de mes fils travaille dans l'art, l'autre est moins passionné mais collectionne aussi.

Q Mais vous même, quelquefois vous n'avez pas envie d'un mur blanc?

R Ah non non, pas du tout !

Q Votre mari fonctionnait sur les coups de coeur, et vous de façon raisonnée. Vous croyez qu'il y a une différence entre une collectionneuse et un collectionneur?

$\mathrm{R}$ Non, je ne pense pas que le fait d'être un homme ou une femme importe, pas le moins du monde... Non, je pense que mon mari est comme ça et que moi je suis comme ça.

Q Les grandes collectionneuses aujourd'hui, à Paris ou en France, collectionnent quoi, comme genre de tableaux ? Est-ce qu'il y a des genres que les femmes préfêrent ? Est-ce qu'elles collectionnent du contemporain?

R Oh ! oui bien sûr. Il y a des collectionneuses de contemporain.

Q Chez les artistes très contemporains, y a-t-il beaucoup de femmes ? Est-ce que les femmes collectionneuses vont plus volontiers vers les femmes artistes? Pensez-vous que le critère du sexe entre en compte?

$\mathrm{R}$ Non, je ne le pense pas. Non, pas du tout...

Q Vous nous avez dit que vous aviez de fortes relations avec Beaubourg, le Musée d'art contemporain ; et avec l'Université ? 
R Oh non ! Je ne cours pas après les conférences, j'en ai fait plusieurs, il y a maintenant une école d'art à Rueil Malmaison, et comme ils savent que nous collectionnons, ils nous ont demandé deux ou trois fois de parler, une fois de César, une fois de la collection. Mais je le fais le moins possible.

Q Que faisaient vos parents?

$\mathrm{R}$ Ma mère n'a jamais travaillé, et mon père était chef des études économiques à la Banque de France.

Q Votre mari était officier, et généralement dans ce milieu on n'aime pas beaucoup que les femmes travaillent.

E Oui, personne ne travaillait à cette époque-là, et puis nous habitions en Algérie et il n'y avait pas beaucoup de possibilités de travail.

Q Vous, vous avez eu une formation très brillante, ce n'est pas donné à toutes les femmes...

R J'étais la seule femme chez Fulmen, il y avait un bâtiment entier pour les bureaux d'études et j'étais l'unique femme. C'était un rêve... je leur faisais même le thé à cinq heures.

Q Après votre mariage, vous n'avez pas travaillé. Mais après votre retour d'Algérie, si vous n'aviez pas été malade, auriez-vous repris le même travail ?

$\mathrm{R}$ Non, j'aurais fait autre chose, quelque chose qui me laisse plus libre. J'avais deux enfants, et je voulais aussi m'occuper d'eux, je n'avais pas envie de rentrer à sept heures du soir. Je n'aurais certainement pas repris un travail très prenant.

$\mathrm{Q}$ À votre retour en France, et quand votre mari a dû quitter l'armée, il avait une idée, lui, de ce qu'il voulait faire?

$\mathrm{R}$ Non pas du tout, cela a été deux ans de grosse déprime.

Q Ce qui l'a engagé dans cette voie nouvelle, c'est l'héritage de la collection?

R Oui bien sûr ! Cet héritage permettait de repartir.

Q Vous avez des petits-enfants?

R Oui, deux petites-filles de 10 et 12 ans.

Q Comment les initiez-vous à votre amour de l'art ?

$R$ Nous faisons des visites de musées, et nous nous baladons dans la maison, je les emmène voir les grandes expositions chaque fois que je peux. Ce ne sont pas des visites de trois heures...

Q Est-ce que vous vous intéressez à la peinture ancienne?

$\mathrm{R}$ Ah ! oui, j'adore. Quand je vais à Venise pour la Biennale, tous les deux ans, je retourne toujours voir les Carpaccio et les Bellini, etc. Je suis alsacienne d'origine, j'habitais à côté de Colmar pendant les vacances, et chaque année évidemment j'allais voir le retable d'Issenheim. La première fois que j'ai emmené mes petites-filles voir le Grünewald, celle de cinq ans a dit : "Ça me dégoûte plutôt », en regardant le Christ allongé sous la Crucifixion...

87 Q Vous vous êtes aussi occupée de la fondation pour les jeunes, la Fondation Garouste. Qu'est-ce que c'est exactement?

E Ah ! La Source est formidable ! La première fois que j'y ai été, j'y suis allée avec César. J'ai tout regardé, c'était un dimanche où les enfants exposaient leurs peintures, et j'ai trouvé cela extraordinaire comme idée, comme action. C'est une fondation qui fonctionne 
grâce au mécénat et à des subventions de l'Éducation nationale et d'autres organismes, et qui est faite pour les enfants en grande difficulté. Ils recueillent tous les enfants qui passent leurs week-ends à traîner dans les rues, c'est en Normandie, à la Guéroulde dans l'Eure. Ils font des ateliers avec chaque fois un artiste, et les enfants font des œuvres. L'atelier ressemble évidemment toujours un petit peu à l'œuvre de l'artiste. C'est absolument merveilleux, les œuvres des enfants sont formidables. J'étais tellement enthousiaste que j'ai dit à Gérard Garouste : "Si jamais tu a besoin de moi, tu me demandes, je suis prête à t'aider ». Je donnais déjà pour des oeuvres, mais je ne faisais rien d'actif. Nous avons fondé avec une amie un cercle des Amis de la Source, et nous essayons de recruter le plus de membres possible chaque année pour faire des dons. Les artistes viennent d'un peu partout, soit le week-end et ils ont des ateliers avec les enfants, soit ils sont résidents et s'occupent des enfants les mercredis, les samedis.

Q Cela a donc à la fois un but pédagogique et une fonction de mécénat vis-a-vis des artistes en résidence?

$\mathrm{R}$ Exactement. Ils sont en train d'ouvrir un deuxième lieu, à Villarceau dans le département voisin. Une fois par an, il y a une grande exposition des enfants de tous les travaux de l'année.

Q Que deviennent les cuvres des enfants ?

R C'est un problème : qu'est-ce qu'on a le droit de faire et qu'est-ce qu'on peut faire avec ces œuvres ? C'est dommage qu'elles s'accumulent dans des hangars et qu'elles ne servent à rien.

Q Finalement, l'idée d'une collectionneuse qui serait enfermée dans son monde, ce n'est pas du tout exact. Faire une collection, pour vous, cela vous met en prise avec le monde social?

R Oui oui, bien sûr, cela m'apporte beaucoup plus que si je passais mes soirées à jouer au bridge avec mes copines. Comme je me retrouve souvent seule, puisque mon mari a émigré à la campagne, seule toute la semaine, cela ne me dérange pas le moins du monde, je descends à Paris, et je vais à droite et à gauche :à un vernissage, retrouver les copains, dîner ensemble, etc.

Q Ensuite, vous pensez que votre collection va être reprise en main par vos enfants de la même manière?

R Oh ! oui bien sûr. D'ailleurs, je vais vous dire, on a déjà fait le partage et une dation à Orsay.

Q Vos fils ont-ils les mêmes goûts?

R L'âné a fait de belles études aux États-Unis et voulait devenir homme d'affaires. De retour en France, il s'ennuie pendant trois ans dans une banque, et me dit : «Tous les gens que je côtoie, c'est dans l'art, la seule chose qui m'intéresse, c'est l'art, je veux travailler dans l'art ». Il a été pendant cinq ans chez Francis Briest, où il a tout appris sur l'art contemporain, et il a aussi fait des études, ensuite il a ouvert sa propre galerie, avec Fabienne Leclerc. C'est lui qui maintenant est chez Christie's. Le second a fait une business school, il travaille depuis comme commercial dans l'aviation, il collectionne, mais avec moins de passion, il est moins impliqué. Il a moins de temps aussi.

$99 \mathrm{Q}$ Le fait de constituer des archives des peintres vous fait devenir un peu spécialiste de l'œuvre de l'artiste.

R Bien sûr, exactement ! Et récemment, il y a eu toute l'histoire des faux César, il y a eu aussi pas mal de faux Arman fabriqués en Italie, maintenant cela devient une maladie. Ils 
me demandent tous mon numéro d'archives, cela me prend un temps fou, à longueur de journée... Il faut arrêter ça.

Q Vous suivez la carrière de vos artistes?

R J'ai pas mal suivi Jean-Pierre Raynaud dans ses expéditions, d'abord parce que ça m'amusait, il a fait des expositions en Chine, au Japon, en Amérique du nord, du sud ; je ne m'occupe pas d'organiser les expositions mais je m'occupe souvent du catalogue. Parce que les photos viennent la plupart du temps de mes archives.

Q Quels sont vos projets maintenant?

$\mathrm{R}$ Eh bien ! là, je vais pouvoir recommencer Arman. J'ai fait seulement deux catalogues, mais je ne vais pas faire toute son œuvre, je vais m'arrêter dans les années 1975. Après, il y en a trop, ça devient des multiples, presque.

$\mathrm{Q}$ Est-ce que ces artistes font aussi des dessins?

$\mathrm{R}$ Ça dépend : Jean-Pierre Raynaud pas du tout, mais Arman oui, César oui. César dessinait beaucoup. Et puis des collages, il adorait faire des petits collages, il bricolait etc. Il avait une main extraordinaire.

Q Qu'est-ce que finalement vous retenez de préférence dans votre collection?

$\mathrm{R}$ Il y a des artistes que j'aime beaucoup personnellement, mais qui n'iraient pas dans notre collection : Soulages, Garouste... Il est impossible de tout collectionner, nous avons fait des choix.

Denyse Durand-Ruel nous montre alors l'endroit où elle travaille sur ses archives, un bureau extraordinaire qui a été conçu par Jean-Pierre Raynaud. Puis elle nous guide à travers sa collection.

\section{NOTES}

1. Le texte de l'entretien a été revu par Denyse Durand-Ruel.

\section{AUTEUR}

\section{DENYSE DURAND-RUEL}

Denyse DURAND-RUEL, mathématicienne, a été ingénieur spécialisée en machines-outils aux usines Fulmen. En 1956, elle épouse Philippe Durand-Ruel ; tous deux s'installent à Paris en 1961 et débutent la constitution d'une collection d'art contemporain. À partir de 1967, Denyse DurandRuel commence un travail d'archivage des œuvres de quatre artistes : Jean-Pierre Raynaud, César, Arman et Bernard Lavier. En 1991, elle publie le premier tome du catalogue raisonné 
d'Arman, en 1994 le second ainsi que le premier tome du catalogue raisonné de César (La Différence) et, en 1998, le premier tome du catalogue raisonné de Jean-Pierre Raynaud (Éditions du Regard). 